Milchevskaya et al.

\title{
Group size planning of breedings of gene-modified animals
}

Vladislava Milchevskaya ${ }^{1,2,{ }^{*}}$, Philippe Bugnon ${ }^{3,}{ }^{\star}$, Emiel B.J. ten Buren ${ }^{3}$, Frank Brand ${ }^{4}$, Achim Tresch $1,2,5, \#$, Thorsten Buch,"\#

${ }^{1}$ Cologne Excellence Cluster on Cellular Stress Responses in Aging-Associated Diseases (CECAD), University of Cologne, Cologne, Germany

Institute of Medical Statistics and Computational Biology, Faculty of Medicine, University of Cologne, Cologne, Germany.

3Institute of Laboratory Animal Science, University of Zurich, Zurich, Switzerland 4Berlin School of Economics and Law, Department of Business and Economics, Quantitative Methods, Berlin, Germany

${ }^{5}$ Center for Data and Simulation Science, University of Cologne, Cologne, Germany

${ }^{*}$,\# contributed equally

\section{Correspondence to:}

Thorsten Buch

Institute of Laboratory Animal Science

University of Zurich

Wagistrasse 12

$\mathrm{CH}-8952$ Schlieren

Switzerland

thorsten.buch@uzh.ch

Keywords: Mendelian genetics, fertility, litter size, stochastics, power calculation, husbandry, 3R Running title: Group size planning of animal breedings

\section{Abstract}

Animal breeding is time-consuming, costly, and affected by stochastic events related to Mendelian genetics, fertility, and litter size. Careful planning is mandatory to ensure a successful outcome using the least number of animals, hence adhering to the 3Rs of animal welfare. We have developed an $\mathrm{R}$ package, accessible also through an interactive website, that optimizes breeding design and provides a comprehensive report suitable for any breeder of genetically defined traits. 
Milchevskaya et al.

\section{Main}

Rodent research and hence breeding has undergone an explosive development. Mouse Genome Informatics counts $47^{\prime} 000$ curated and 14'000 non-curated entries for mouse lines ${ }^{1}$. A total of 7 Mio rodents per year are used for creation and maintenance of genemodified lines in the European Union alone ${ }^{2}$. In most projects not only single mutants are bred and analyzed: rather combinations of multiple alleles of different genes have become mainstay in research. These complex genotypes are assembled through time and cost intense breeding schemes ${ }^{1}$.

A

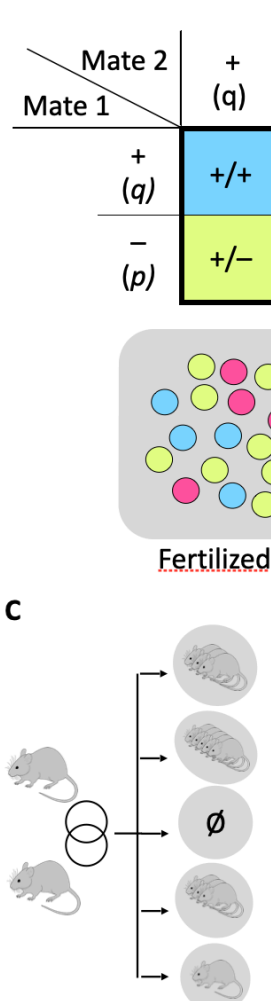

Viable offspring frequencies
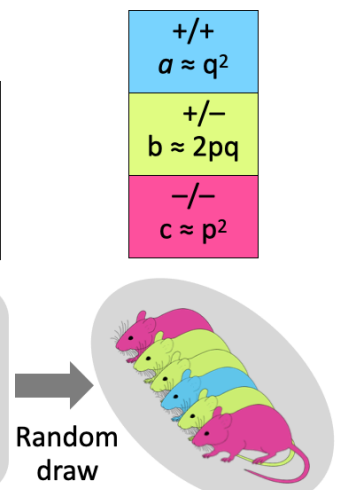

Bred offspring (M)

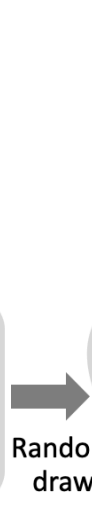

(p)
Offspring number

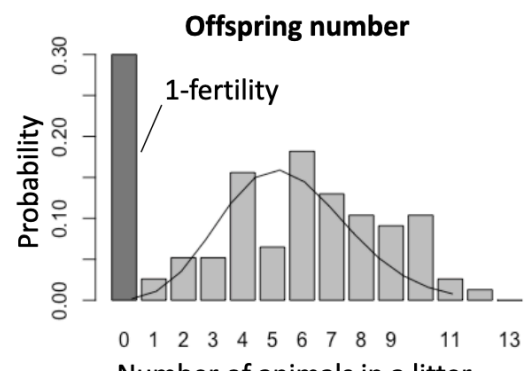

B Number of mice of desired genotype

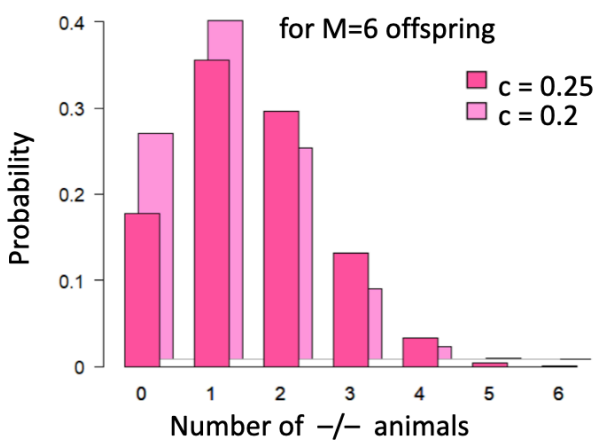

D

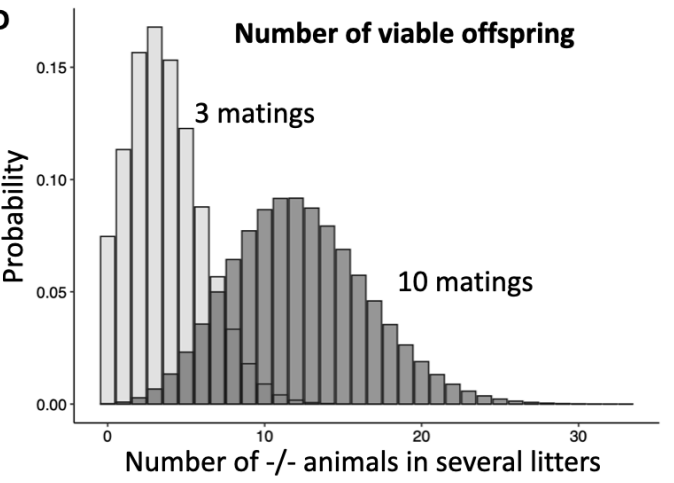

Fig. 1 Stochastic Effects in Breeding. (A) Mendelian model of the outcome of a single breeding of two heterozygous animals. + and - denote different gene alleles occurring with probabilities $q$ and $p$, correspondingly. Theoretical frequencies of the $+/+,+/$ - and $-/-$ offspring are $q^{2}, 2 p q$ and $p^{2}$. An outcome of single breeding is depicted as a random draw from the theoretical distribution above. (B) Probability that out of 6 offspring exactly $X$ animals will have the desired -/- genotype, given the probability $p^{2}$ of the -/- genotype is 0.25 or 0.2 . (C) Example of the number of animals born out of a single breeding when fertility is taken into account: There is a non-zero chance that 0 animals are born (1-fertility). (D) Distribution of -/- animals born for a genotype frequency of 0.25 , for 10 (grey) respectively 3 (light grey) breedings. 
Milchevskaya et al.

The practice of killing animals because they do not carry specific traits or are not needed has become under scrutiny ${ }^{3,4}$, in laboratory animal science 5 , farming ${ }^{6,7}$, and zoos ${ }^{8-11}$. The causes of unwanted surplus in laboratory animal facilities were identified, among them: genetics of breeding, sex preference and the inability to match supply with demand 12 . Stochastic fluctuations in allele distribution, in fertility (some breeding pairs will produce no offspring), and litter size (number of pups born/weaned per litter) have a large influence on breeding outcomes. Their neglect results in unnecessary breeding delays and scientifically unjustified animal use. We describe here software that enables researchers to plan their breedings with a given success probability, integrating the stochastic effects caused by Mendelian genetics, fertility and litter size. Typically, genotype frequencies in offspring are obtained via Punnett square ${ }^{13}$ (Fig 1A). However, in a single or few litter(s), the observed frequencies may differ substantially due to random fluctuations. Given a fixed litter size, the number of e.g. -/- animals being present in a litter from $+/$ - parents follows a Binomial distribution (Fig 1B, Mendelian probability $\mathrm{c}=0.25$ ). If the breeding outcome does not follow classical Mendelian frequencies (e.g. embryonal deaths ${ }^{14,15}$ ), the probabilities of occurrence change (Fig. 1B, $c=0.2$ ). Furthermore, litter size itself is a variable that can either be positive (number of offspring in a litter when the mating is successful) or turn zero when the mating is unsuccessful. We collected the empirical distributions of litter sizes for 8 mouse lab strains and found that most of them could be approximated well by a Poisson distribution (Figure 1C, Suppl. Figure 1), when unsuccessful matings are excluded. The fraction of successful matings (fertility) in our calculator is obtained from the values reported by the Jackson lab (http://www.informatics.jax.org/silver/tables/table4-1.shtml). Having specified these components, we derived the distribution of the target offspring number as a function of the number of matings (Figure 1D, Supplementary Methods). The probability of successfully obtaining the desired number of pups of needed genotypes from a specific setup can then be quantified. In the 1980s, Festing proposed a method for modelling the probabilistic outcomes in fertility and litter size ${ }^{16}$. We developed an alternative method that can be proven more accurate (Supplementary Methods) and thereby often reduces the number of matings to be set up (Fig 2A). We also show that the simplistic use of the expected target animal number derived from Mendel's laws combined with average litter size underestimates the required number of matings dramatically (Fig. 2A, Mendel). We recommend setting the desired success probability not overly high (e.g. below 0.95) since further confidence increase is costly in terms of additional matings. (Figure 2B, Suppl. Figure 3).

Often multiple genotypes need to be produced by the same set of breeding pairs, e.g., identical numbers of $+/+$ and $-/$ - animals from $+/$ - parents. Such a setup reduces success probability further and requires additional matings (Figure $2 \mathrm{C}$ ). The same calculations apply to group size planning for obtaining defined numbers of animals of either sex. While some experimental designs require all animals to be of the same sex, alternative designs can 
Milchevskaya et al.

include both sexes (and account for sex-specific effects)17. A group size planning for inclusion of both sexes at identical number increases the required matings only slightly over simple use of all males and females born, without fixed ratio (Figure 2D). However, the use of only one sex increases the required number of matings massively ${ }^{17}$ (Figure 2D).

A
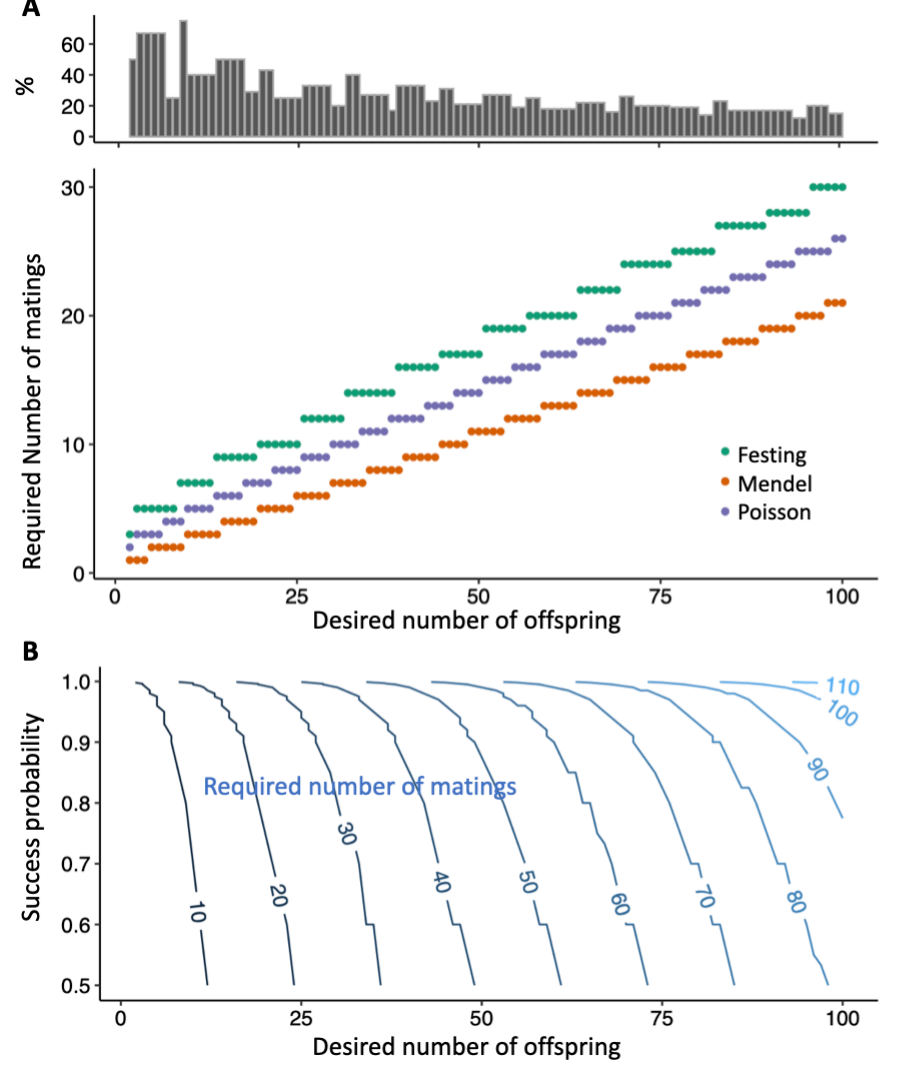

C

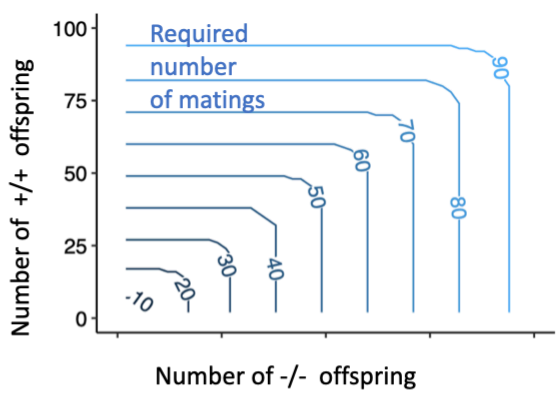

D

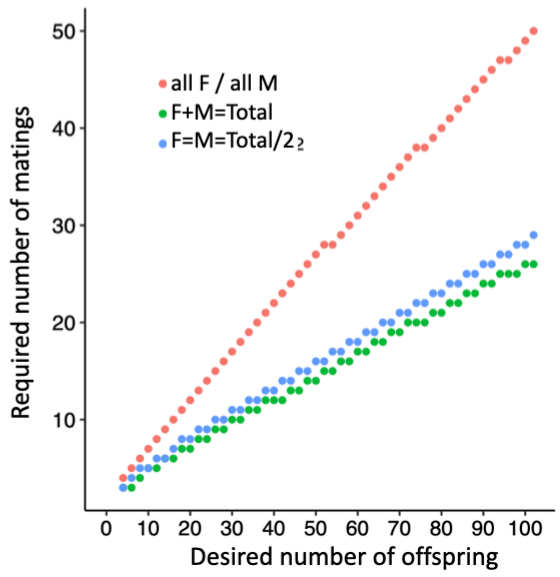

Figure 2. Performance of breeding models, in the case of an average litter size of 7 and a mouse fertility of $\mathbf{7 0 \%}$. (A) Bottom: Minimal required number of breedings ( $Y$-axis) needed to obtain the desired number of offspring (X-axis) with $90 \%$ confidence, as calculated by three methods: The naïve expectation due to Mendelian frequencies (see Suppl.), the gold standard textbook model suggested by Festing, and our method, denoted as Poisson. Top: Relative surplus of breedings (in \%) required by the textbook method (Festing), measured against our method. (A, bottom)

(B) Minimal number of breedings needed to obtain a certain number of offspring (X-axis) with a defined probability of success (Y-axis). (C) Minimal number of breedings (contour lines) required in a setup where groups with two different genotypes need to be produced by the same breedings. (D) Minimal number of breedings required to obtain offspring of specific sexes with $90 \%$ confidence, given that both female $(F)$ and male $(M)$ pups are born with equal probability. Shown are three scenarios: $X$ offspring required to be of the same sex required (red), $X$ offspring of any sex required (green), $X$ offspring with balanced cohorts of each sex required, i.e. $X / 2$ male and $X / 2$ female pups (blue). 
Milchevskaya et al.

To facilitate appropriately powered breeding for the practitioner, we incorporated the algorithms and data for sample size calculation into the $\mathrm{R}$ package 'BreedingCalculator', available at the GitHub repository https://github.com/VladaMilch/breedingCalculator. Simplified, interactive access to this package is provided on the website https:// www.ltk.uzh.ch/en/Breeding.html. We complemented the package with an additional parameter, namely effective fertility, based on the numbers given by Festing ${ }^{16}$. Effective fertility comes into effect when the age of the experimental cohort is fixed to a short time period such as birth within 1,2 or 3 days ${ }^{16}$.

Optimization of breeding protocols for reduction of animal use is an ethical obligation mandated within the commonly applied $3 R$ (replace, reduce, refine) principle. Yet, the very basic biology of mammalian genetics and associated stochastic processes inevitably create surplus animals without further use in either experiments or breeding. We have developed an $\mathrm{R}$ package that supplies the optimal solution, i.e. the least number of required animals, for every use case described above. It uniformly improves over previously published tables and schemes (e.g. , https://www.jax.org/jax-mice-and-services/customer-support/manualsposters-and-guides/jmcrs-manuals-guides). We removed from the workflow any form of guess work commonly done by scientists to adjust for self-experienced stochastic effects, colloquially referred to as Murphy's law. Also, through appropriate group size calculations for breedings, experiments are more likely to be conducted as planned, which serves their reproducibility. It may seem that powered breeding planning increases the number of animals produced for an experiment. But this is not the case, because if the planning is not adequate and the desired number of animals is not reached, a new breeding round will be required and the animals from the first round remain unused. Hence, correct planning allows in a precise way to reach the required number of animals by reducing the number of unsuccessful breeding attempts. While unequal use of the sexes in animal experimentation has been discussed thoroughly, with statistical solutions regarding experimental designs suggested, we here provide evidence that restricting experiments to one sex unnecessarily leads to additional breedings and hence unused offspring beyond a simple doubling.

Taken together, we provide a method supported by software to minimize animal numbers in breeding while explicitly controlling for experiment's success, being in full compliance with the $3 \mathrm{R}$ rule. 
Milchevskaya et al.

\section{References}

1. Bult, C. J., Blake, J. A., Smith, C. L., Kadin, J. A. \& Richardson, J. E. Mouse Genome Database (MGD) 2019. Nucleic Acids Research 47, D801-6; 10.1093/nar/gky1056 (2018).

2. European Commission. on the implementation of Directive 2010/63/EU on the protection of animals used for scientific purposes in the Member States of the European Union. Report from the commission to the European parliament and the council, 5. February 2020.

3. Lewejohann, L., Grune, B., Schönfelder, G. \& Bert, B. Cut back on surplus laboratory animals. Nature 578, 515; 10.1038/d41586-020-00517-3 (2020).

4. Expose Animal Experiments. Outlaw the killing of "surplus" animals bred for experiments \& enforce their compulsory rehoming (2020).

5. Doehring, D. \& Erhard, M. H. Verbleib von überzähligen und überlebenden Versuchstieren. ALTEX 22, 7-11 (2005).

6. Royal Society for the Prevention of Cruelty to Animals. What happens with male chicks in the egg industry? (2020).

7. Vizzier Thaxton, Y. et al. Symposium: Animal welfare challenges for today and tomorrow. Poultry science 95, 2198-2207; 10.3382/ps/pew099 (2016).

8. Performing Animal Welfare Society. Surplus animals: the cycle of hell. A Study of Captive Wildlife in the United States. Available at https://www.pawsweb.org/surplus.pdf.

9. Hannah Barnes. How many healthy animals do zoos put down? BBC News (2014).

10. Ian Parker. Killing animals at the zoo. Newyorker (2017).

11. R. Scott Nolen. Zoos wrestle with fate of surplus animals. Available at https:// www.avma.org/javma-news/2002-12-01/zoos-wrestle-fate-surplus-animals (2002).

12. Lasa task force meeting. The production and disposition of laboratory rodents surplus to the requirements for scientific procedures. Available at http://www.lasa.co.uk/PDF/ Surplus.pdf (1998).

13. Punnett, R. C. Mendelism (Wilshire, New York, 1909).

14. Li, X. et al. A Maternal-Zygotic Effect Gene, Zfp57, Maintains Both Maternal and Paternal Imprints. Developmental cell. 15, 547-557; 10.1016/j.devcel.2008.08.014 (2008).

15. Kallapur, S., Ormsby, I. \& Doetschman, T. Strain dependency of TGF beta 1 function during embryogenesis. Molecular reproduction and development. 52, 341-349 (1999).

16. Festing, M. Animal production and breeding methods. UFAW handbook on the care and management of laboratory animals 6th edition (1987).

17. Buch, T. et al. Benefits of a factorial design focusing on inclusion of female and male animals in one experiment. Journal of molecular medicine (Berlin, Germany) 97, 871877; 10.1007/s00109-019-01774-0 (2019). 
bioRxiv preprint doi: https://doi.org/10.1101/2021.09.17.460764; this version posted September 20, 2021. The copyright holder for this

preprint (which was not certified by peer review) is the author/funder, who has granted bioRxiv a license to display the preprint in perpetuity. It is made available under aCC-BY-NC 4.0 International license.

Milchevskaya et al.

18. The Jackson Laboratory. Breeding Strategies for Maintaining Colonies of Laboratory Mice. A Jackson Laboratory Resource Manual (2009).

19. Flórez-Vargas, O. et al. Bias in the reporting of sex and age in biomedical research on mouse models. eLife 5; 10.7554/eLife.13615 (2016).

20. Itoh, Y. \& Arnold, A. P. Are females more variable than males in gene expression? Metaanalysis of microarray datasets. Biology of sex differences 6, 18; 10.1186/

s13293-015-0036-8 (2015). 
Milchevskaya et al.

\section{Supplementary Figures}

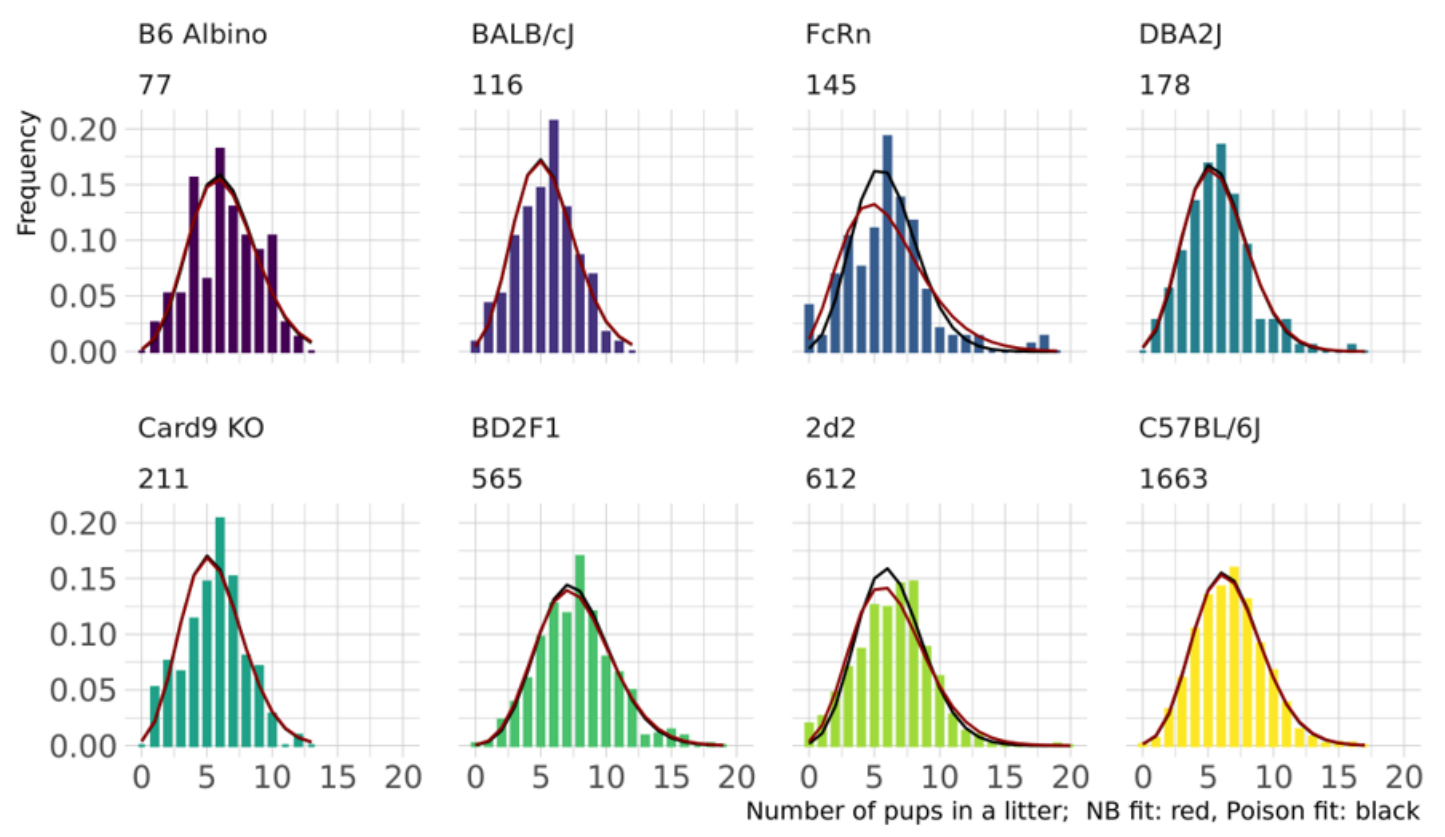

Figure S1: Litter size distribution for different mouse strains, estimated from the corresponding number of litters (under the strain name). On top of each histogram, fits of the Negative Binomial (red) and Poisson (black) distributions are displayed. The fits were performed using the Maximum Likelihood approach implemented in the MASS package in R. Strain/line names are indicated and refer to: B6 Albino - B6N-Tyrc-Brd/BrdCrCrl, BD2F1 - B6D2F1, Card9 KO - C57BL/6N-Card9 ${ }^{\mathrm{em} 11 \mathrm{tk}}$, FcRn - C57BL/6.CgFegrt $^{\text {tm1Der }}$ Tg(FCGRT)32Dcr/DcrJ, 2d2 - Tg(Tcra2D2,Tcrb2D2)1Kuch, total number of used litters is indicated under the line name. 
Milchevskaya et al.

\section{Median number of born aminals for a setup with average litter 7 and average fertility $70 \%$}

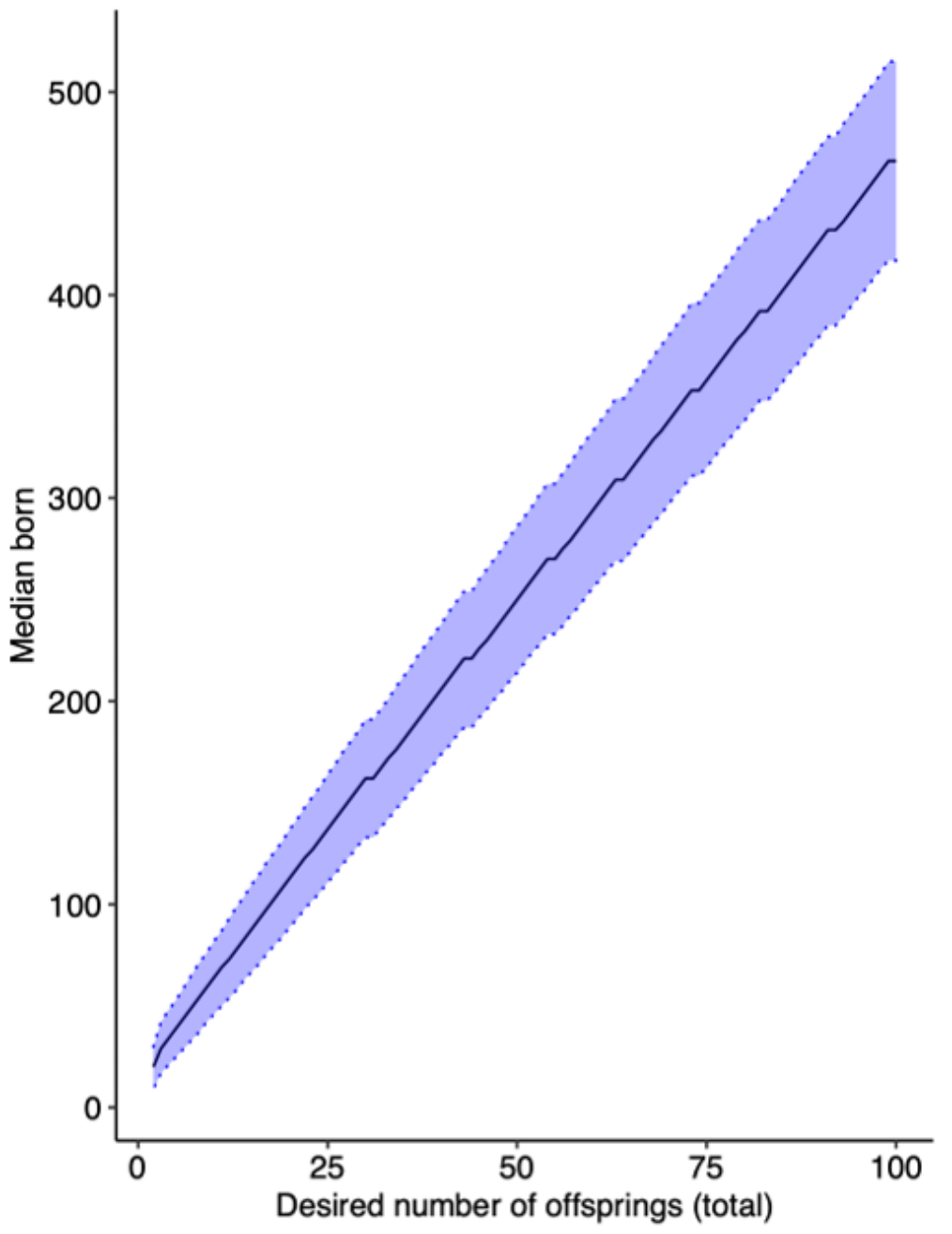

Figure S2: Total number of born animals, when the breeding setup is designed to yield no less than $X$ animals with success probability $90 \%$. Blackline depicts the median number of animals born, the blue area around corresponds to the symmetric confidence interval of 0.8 . 
Milchevskaya et al.

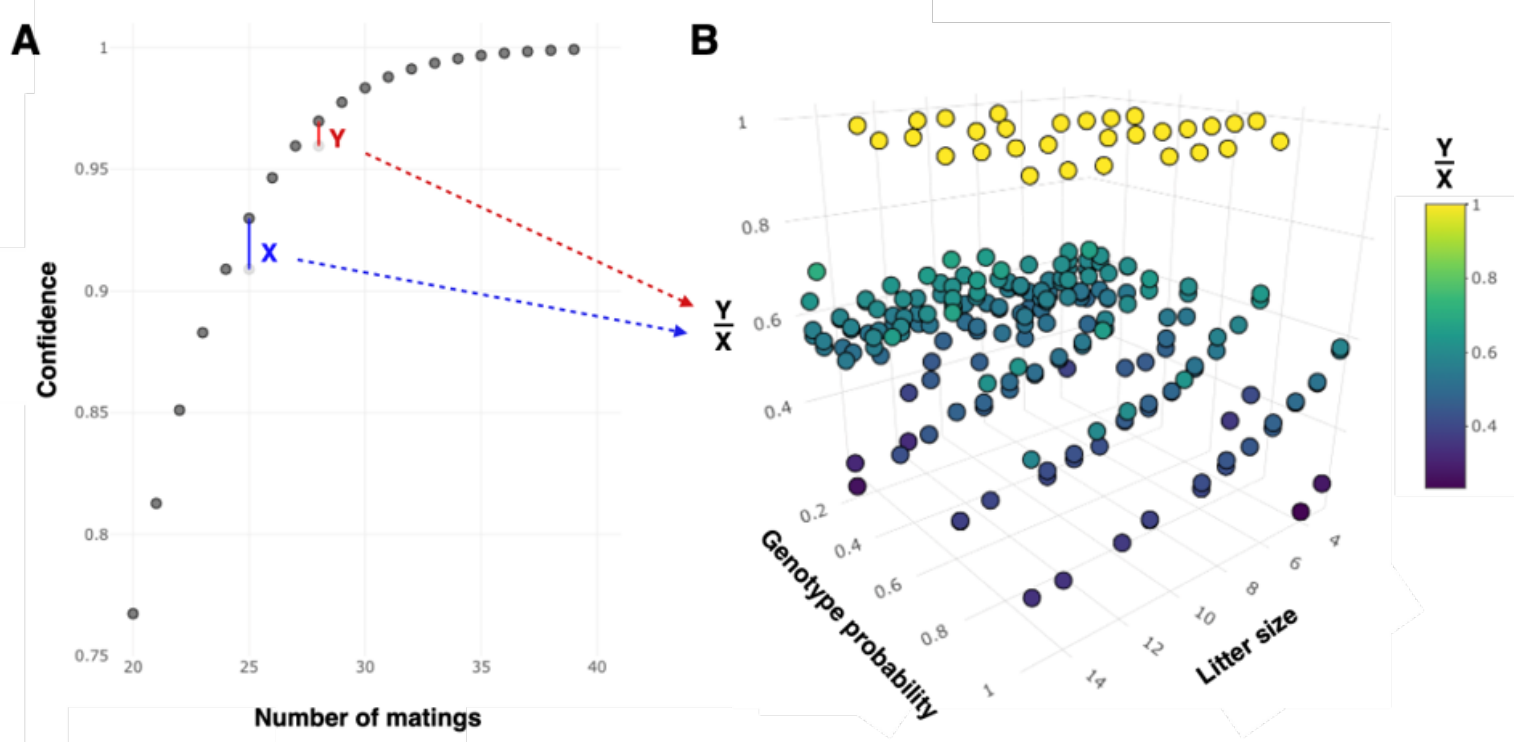

Figure S3: Optimal confidence level choice. Panel A shows an example of the relation between the number of matings and the confidence in the successful outcome of the breeding; segments $X$ (blue) and $Y$ (red) show the confidence increase granted by one additional mating: $X$ - starting from the number of matings sufficient for the $90 \%$ confidence, $Y-$ from the number of matings sufficient for the $95 \%$ confidence. One can see that the higher the target confidence, the smaller the confidence increase - which is especially pronounced for the high confidence values (flat curve). The parameters used for this example: the average litter size of 5 , fertility of $50 \%$, desired genotype probability of $50 \%$, the desired number of pups of this genotype - 20. Panel B shows the ratio of the $95 \%$ and the $90 \%$ confidence increases from panel A, calculated for different starting parameters for the litter size, the fertility (not shown) and the desired genotype probability. Hence, one point corresponds to a single example as in panel $\mathrm{A}$.

For some cases (yellow), when very few matings are required to yield a successful breeding, there is no difference between $X$ and $Y$, thus the ratio is 1 . However, for the majority of breeding parameters, the increase in confidence above from $95 \%$ is from 1.5 to 10 fold smaller than the increase from $90 \%$ (Y/X values below 0.6 ). We therefore recommend choosing the target confidence level not too high (often, below 95\%). 